Insgesamt ergaben die beschriebenen Dressurversuche, daß die Druckrezeption auch bei Orientierungsleistungen mitwirken kann, jedoch nur eine begrenzte Rolle dabei spielt. Wahrscheinlich kann ein Schwimmblasenfisch außer Druckänderungen noch registrieren, ob er sich oberhalb oder unterhalb der Äquilibrationsebene befindet und wieweit er ungefähr davon entternt ist. Die Mechanismen der Druckrezeption (Schwimmblase - Weberscher Apparat) erscheinen nicht geeignet, einen Druck seiner absoluten Größe nach zu regi- strieren und dem Fisch ein Rezeptionsvermögen für die Wassertiefe $z$ u geben.

Zoologisches Institut der Universität, Köln (Divektor: Prof. Dr. Otro KunN) Eberhard Strotkoetter

Eingegangen am 15. September 1960

1) BAglioni, S.: Z. allg. Physiol, 8, 1 (1908). - 2) DijkgraAf, S.: Z. vergl. Physiol. 28, 389 (1941); 30, 39 (1942). - 3 ) MoEHREs, F.P.: Z. vergl. Physiol. 28, 1 (1941)

\title{
Bespredhungen
}

Stellar Populations. Proceedings of the Conference sponsored by the Pontifical Academy of Science and the Vatican Observatory. Hrsg. von D. J. K. O'Connell, S. J. Amsterdam: North Holland Publish. Comp.; New York: Interscience Publishers, Inc. 1958. LXVII, 544 S., 3 Bilder u. 57 Fig. Gr.-8. Gzl. Gulden 38.-

In einer 1944 publizierten Arbeit über den Andromedanebel und dessen elliptische Begleiter wies BAADE darauf hin, $\mathrm{da} \beta$ in Galaxien zwei Sternpopulationen vorhanden sein können, die sich empirisch vor allem in ihrer räumlichen Verteilung und in ihrem Farben-Helligkeitsdiagramm unterscheiden: Die Population der Spiralarme, die Baadesche Population I, und eine Population II, aus der die elliptischen Nebel bestehen und die in den Kerngebieten der Spiralsysteme anzutreffen ist. Diese Entdeckung ist für die Astrophysik außerordentlich bedeutsam geworden: Wir wissen heute, daß die beiden Populationen zwei Sternsorten verschiedenen Alters repräsentieren; die Sterne der Population I sind junge Sterne, während wir bei der Population II alte Objekte vor uns haben. BAADEs Beobachtungen bilden dadurch die Grundlage für die heutigen Vorstellungen über die zeitliche Entwicklung der Sterne und Sternsysteme. - Das vorliegende Buch berichtet über ein 1957 an der Vatikan-Sternwarte veranstaltetes internationales Symposium, das, mit etwa zwanzig Experten als Teilnehmern, ausschließlich dem Problemkreis der Sternpopulationen gewidmet war. Als wichtiges Ergebnis der Konferenz sei die Verfeinerung des Populationsbegriffs hervorgehoben. BAADEs Populationen I und II sind heute als die beiden Extreme einer Sequenz anzusehen, deren physikalische und kinematische Zustandsgrößen sich mit dem Alter der Objekte kontinuierlich ändern. In 34 Beiträgen einschließlich der Diskussionsbemerkungen findet der Leser eine praktisch erschöpfende Behandlung der so verschiedenartigen Aspekte des Themas. Der Stoff gliedert sich im wesentlichen in die drei Abschnitte: Typische Objekte der beiden Populationen, chemische Zusammensetzung der Populationen und Sternentwicklung, räumliche Verteilung und kinematisches Verhalten der Sternpopulationen im Milchstraßensystem. Eine Reihe von Übersichtsreferaten ermöglicht auch dem Nichtspezialisten das Verständnis und gibt dem Bericht den Charakter eines Lehrbuches. Dem Fachmann wie dem Fernerstehenden sei es als Information aus erster Hand über eines der aufregendsten Arbeitsgebiete der heutigen Astrophysik wärmstens empfohlen.

H. ExSÄSSER (Göttingen)

Noller, c. R.: Lehrbuch der organischen Chemie. Übersetzt von H. MaYer-Kaupp u. P. Stephan. Berlin-Göttingen-Heidelberg: Springer 1960. VIII, 1018 S. u. $106 \mathrm{Abb}$. Gr.-8 ${ }^{\circ}$. Gz1. DM 36.-

Das Lehrbuch der organischen Chemie von C. R. NoLler hat bereits in der amerikanischen Originalausgabe Eingang in den deutschen Hochschulunterricht gefunden. Es wird in der gut gelungenen Übersetzung, in einiger Hinsicht unseren Verhältnissen angepaßt, schnell an Verbreitung zunehmen. Der Erfolg des Buches beruht darauf, daß es ein erstaunlich großes Tatsachenmaterial in systematischer Ordnung bringt und gleichzeitig die theoretischen Vorstellungen erläutert und begrïndet. Hierin geht es weiter als die meisten elementaren Lehrbücher bei uns. Gleich im Anfang wird die Theorie der chemischen Bindungen so anschaulich wie möglich aus den Elektronenzuständen der Atome hergeleitet. Das gibt später die Möglichkeit, Reaktionsmechanismen zu diskutieren und die Wirkungsweise der Katalysatoren zu verstehen. Bei den Carbonsäuren kommt der Begriff der Resonanz hinzu, unentbehrlich vor allem in der aromatischen Reihe. Die Theorie der Farbstoffe wird durch eine Übersicht über die Spektren eingeleitet; damit wird auch die Bedeutung der UV-, IR- und Kernresonanzspektroskopie für die Konstitutionsermittlung verständlich. Neben dem roten Faden der Theorie kommt das Stoffliche keineswegs zu kurz. Die Entdeckungsgeschichte wichtiger Substanzen sowie eingehende Schilderungen ihrer Technologie und Bedeutung zu verschiedenen Zeiten geben das lebensvolle Bild einer Wissenschaft und Technik, bei der alles in ständigem Fluß ist. Auch der bedeutendsten Forscher wird dabei gedacht. Der deutsche Leser wird vielleicht die Behandlung der Heterocyclen allzu kurz finden und die Biochemie vermissen.

Das Buch ist für Anfänger geschrieben. An jedes der 42 Kapitel ist eine Sammlung von Wiederholungsfragen und Aufgaben angeschlossen, die man als recht schwierig bezeichnen muß. Thre vollständige Durcharbeitung dürfte dem Anfänger nur mit Hilfe einer Lehrperson möglich sein.

Im ganzen aber liest sich das Buch sehr gut und überascht selbst den fertigen Organiker mit einer Fülle wenig bekannter Angaben und Hinweise. Der Verlag hat den Band gut ausgestattet und den Preis bemerkenswert niedrig gehalten. Die Anschaffung kann allen Studenten der Chemie und Pharmazie warm empfohlen werden.

\section{G. HESSE (Erlangen)}

Wollman, E. L. et F. Jacob: La Sexualité des bactéries. Paris: Masson Cie. 1959. 248 S., 27 Abb. u. 23 Tab. Gr.- $8^{\circ}$. Brosch. $3000 \mathrm{Fr}$.

Die beiden Autoren legen mit ihrem Buch die klar geschriebene Zusammenfassung von experimentellen Ergebnissen vor, die, aufbauend auf ersten Befunden von LEDERBERG und TATUM sowie Hayes über sexuelle Vorgänge bei Bakterien, in relativ kurzer Zeit von ihnen selbst erarbeitet und zu einem übersichtlichen Bilde der hierbei obwaltenden Mechanismen zusammengefügt werden konnten. Der Leser bekommt also aus erster Hand, d.h, ohne Mißverständnissen von seiten menschenfreundlicher Referenten ausgesetzt zu sein, einen tiefen Einblick in ein ganz neu erschlossenes Kapitel genetischer Forschung. Man darf den beiden Meistern ihres Faches wirklich dankbar sein, daß sie sich der Mühe einer zusammenfassenden Darstellung selbst unterzogen haben.

Bakteriengenetik umfaßt eine ganze Reihe ungewöhnlicher biologischer Prozesse, deren Aufklärung und experimentelle Beherrschung nicht nur zunächst unverständliche Kreuzungsergebnisse vollkommen begreiflich macht, sondern darüber hinaus zu neuen Konzepten führt, wie z. B. dem des ,Episoms“ als $Z$ wischenglied zwischen rein cytoplasmatischen und rein chromosomalen Zellelementen, die das Verstehen normaler sowic pathologischer Leistungen der lebenden Zelle zweifellos sehr fördern werden. Es sei deshalb ausdrücklich gesagt, daß die Monographie von Woldman und JACOB keinesfalls nur Spezialisten angeht. Der darin gebotene Stoff ist vielmehr vollkommen unterrichtsreif und für die Biologie so wesentlich, $\mathrm{daB}$ er allen Studierenden der Biologie und Biochemie als unentbehrliches Rüstzeug vermittelt werden müßte.

W. WeIDEL (Tübingen)

\section{Berichtigung}

zu der Kurzen Originalmitteilung "Neue Alkaloide aus Nerine flexuosa und Nerine bowdenii“ von H.-G. Bort und W. DöPkE, Naturwissenschaften 47, 109 (1960): In Absatz 3, Zeile 1 muß es hinter ,Neflexin" heißen: $\mathrm{C}_{18} \mathrm{H}_{21} \mathrm{NO}_{5}$ (nicht $\mathrm{O}_{3}$ )

Herausgeber und verantwortlicher Redakteur: Professor Dr, Ernst Lamla, Göttingen. - Verantwortlich für den Anzeigenteil: Günter Holtz, Berlin-Wilmersdorf, Herausgeber und verantwortlicher Redakteur: Professor Dr, Ernst Lamla, Götingen. - Verantwortlich für den Anzeigenteil: Günter Holtz, Berlin-Winger 